\title{
Anterior Cingulate and Motor Network Metabolic Impairment in Progressive Supranuclear Palsy
}

\author{
E. Salmon, ${ }^{*}$ M. Van der Linden, $\dagger$ and G. Franck* \\ * Cyclotron Research Centre and Department of Neurology and $\uparrow$ Department of Neuropsychology, University of \\ Liège, 4000 Liège, Belgium
}

\begin{abstract}
Progressive supranuclear palsy is the prototype of subcortical dementia. Using positron emission tomography and statistical parametric mapping, we compared the glucose metabolic pattern obtained in this subcortical dementia to that observed in elderly healthy controls and in Alzheimer's disease, the prototype of cortical dementia. Progressive supranuclear palsy was characterized by a relative decrease of metabolisminanterior cingulate, adjacent supplementary motor area, precentral cortex, middle prefrontal cortex, midbrain tegmentum, globus pallidus, and ven-trolateral and dorsomedial nuclei of thalamus. The data in progressive supranuclear palsy highlight predominant metabolic impairment in brain structures engaged in response selection, in attention for action, and in motor networks.
\end{abstract}

\section{INTRODUCTION}

The term "subcortical dementia" was introduced to describe a clinical syndrome observed in progressive supranuclear palsy (PSP) and characterized by forget-fulness, slowing of thought processes, emotional or personality changes, and impaired ability to manipulate acquired knowledge (Albert et al., 1974). Other conditions such as Huntington's disease and Parkinson's disease have also been labeled subcortical dementia. In the absence of aphasia, apraxia, and agnosia, the subcortical dementing pattern was contrasted to that occurring in cortical dementia such as Alzheimer's disease (Albert et al., 1974). However, the distinction between cortical and subcortical dementia is a matter of debate; both distinguishing as well as overlapping neuropsychological and neuropathological patterns are reported in the literature (Whitehouse, 1986; Pillon et al., 1991; Hauw et al., 1990; Gearing et al., 1994). Characteristic patterns of cerebral blood flow and metabolism have been described in degenerative dementias using positron emission tomography (PET) or single-photon emission tomography, but the precise differences and similarities between cortical and subcortical dementias have rarely been specifically addressed (Habert et al., 1991; Goto et al., 1993).

The metabolic pattern observed in PSP using PET and regions of interest analysis is characterized by combined cortical (mainly frontal) and subcortical involvement (D'Antona et al., 1985; Leenders et al., 1988; Foster et al., 1988; Goffinet et al., 1989; Blin et al., 1990). However, new image-processing technology allows analysis of all available metabolic information, without a priori hypotheses based on anatomic regions of interest (Friston et al., 1995). Such analyses recently emphasized a predominance of metabolic impairment in posterior cingulate cortex among the associative posterior regions typically involved in Alzheimer's disease (Minoshima et al., 1994; Reiman et al., 1996).

Using statistical parametric mapping (Friston et al., 1995), we contrasted the glucose metabolic patterns obtained with PET in progressive supranuclear palsy to that observed in healthy controls with similar age, and inAlzheimer's disease of the senile type (SAD) with mild dementia, to emphasize the specific brain areas functionally involved in the disease. We observed in PSP a predominance of metabolic impairment in subcorticofrontal motor and cognitive networks, the latter comprising anterior cingulate cortex which is a key structure in some executive functions (Devinsky et al., 1995).

\section{MATERIALS AND METHODS}

\section{Subjects}

The metabolic pattern of glucose utilization was studied in 10 patients (mean age 67.865 .9 years) with a clinical diagnosis of progressive supranuclear palsy (Steele et al., 1964; Daniel et al., 1995; Collins et al., 1995), 12 patients (mean age 69.3 64.6 years) with probable Alzheimer's disease (McKhann et al., 1984), and 8 elderly healthy volunteers (mean age 63.466 .1 years). All patients with PSP had supranuclear down-gaze paresis, postural instability, bradykinesia, axial rigidity, nonblinking facies, dysarthria, and poor response to dopatherapy. None had dopaminergic treatment at PET time, and parkinsonian score ranged from 13 to 45 on the King's College Parkinson's disease scale (Parkes et al., 1970; Table 1). All patients met NINDS- SPSP clinical research criteria for the diagnosis of probable PSP (Litvan et al., 1996). All patients with AD fulfilled the DSMIII-R criteria for dementia (American Psychiatric Association, 1987) and most of them were of the SAD type (Table 2). The diagnosis, according to widely accepted criteria (McKhann et al., 1984), was based on general 
medical, neurological, and neuropsy-chological examination and normality of laboratory tests. PSP patients had questionable to mild dementia, so that SAD patients with mild dementia were selected (Hughes et al., 1982); mean disease duration was shorter in SAD (1.6 year) than in PSP (3.9 years). CT scan showed, at most, mild atrophy. All subjects and a relative gave informed consent to take part in the study, which was approved by the University of Liège hospital ethics committee.

TABLE 1: Demographic and Clinical Data for Patients with Progressive Supranuclear Palsy

\begin{tabular}{lllll} 
Patient & Age (years) & Duration (years) & P score (max 93) & CDR (max 3) \\
\hline 1 & 68 & 2 & 29 & 1 \\
2 & 65 & 4 & 27 & 1 \\
3 & 75 & 1 & 40 & 1 \\
4 & 11 & 41 & 0.5 \\
5 & 75 & 3 & 13 & 0.5 \\
6 & 58 & 3 & 30 & 1 \\
7 & 65 & 3 & 26 & 1 \\
8 & 74 & 2 & 17 & 0.5 \\
9 & 66 & 7 & 18 & 1 \\
10 & 61 & 3 & 45 & 0.5
\end{tabular}

Note. P score, parkinsonian score, is calculated from Parkes et al. (1970). CRD, clinical dementia rating scale (Hughes et al., 1982): 0.5 is questionable, 1 is mild.

\section{PET Image Acquisition and Analysis}

Scans were obtained during quiet wakefulness with eyes closed, on a Siemens 951/31R tomograph (CTI, Knoxville, TN) with collimated septa extended, using the $\left[{ }^{18} \mathrm{~F}\right]$ fluorodeoxyglucose technique (Phelps et al., 1979). A transmission scan was acquired for attenuation correction using three rotating sources of $68 \mathrm{Ge}$. Emission scans were reconstructed using a Hanning filter at a cutoff frequency of $0.5 \mathrm{~Hz}$, giving a transaxial resolution of 8.7-mm full width at half maximum (FWHM) and an axial resolution of $5 \mathrm{~mm}$ FWHM for each of 31 planes, with a total field of view of $10.8 \mathrm{~cm}$ in the axial direction.

PET scans were analyzed by using statistical parametric mapping (SPM 95, Wellcome Department of Cognitive Neurology, London, UK; Friston et al., 1995), in Matlab (Math Works, Natick, MA). For each scan the 31 transverse planes were interpolated to 43 planes to render the voxels approximately cubic. Images were subsequently normalized into a standard stereotactic anatomical space (Talairach and Tournoux, 1988) and were smoothed using a Gaussian filter (12-mm FWHM) to accommodate intersubject differences in gyral and functional anatomy and to increase the signal to noise ratio in the data set. Differences in global metabolism between subjects were removed by analysis of covari-ance (ANCOVA), on a pixel by pixel basis, using the global mean as covariate (Friston et al., 1991). For each pixel in the stereotactic space, the ANCOVA generated an adjusted mean value of cerebral metabolic rate of glucose and an associated adjusted error variance. Significant differences between groups were estimated on a pixel by pixel basis using the $t$ statistic and age as covariate. The resulting sets of $t$ values constituted the statistical parametric map SPM( $t$ ) (Friston et al., 1991). The $\operatorname{SPM}(t)$ was transformed to the unit normal distribution to give a $\operatorname{SPM}(Z)$. We used a $\operatorname{SPM}$ with a $Z$ score threshold of 3.09 ( $P<0.001$, uncorrected for multiple comparisons). Decrease of metabolism was then characterized in terms of probability that a brain area of the observed number of pixels could have occurred by chance over the entire volume analyzed ( $P$ value $<0.05$, corrected for multiple comparisons). The table displays the location of pixels with the maximal $Z$ values comprised in these brain areas. 
TABLE 2: Demographic and Clinical Data for Patients with Alzheimer's Disease

\begin{tabular}{lllll} 
Patient & Age (years) & Duration (years) & MMSE (max 30) & CDR (max 3) \\
\hline 1 & 64 & 1 & 23 & 1 \\
2 & 77 & 1 & 28 & 1 \\
3 & 1 & 23 & 1 \\
4 & 60 & 1 & 23 & 1 \\
5 & 69 & 2 & 26 & 1 \\
6 & 69 & 3 & 23 & 1 \\
7 & 71 & 1 & 23 & 1 \\
8 & 74 & 3 & 23 & 1 \\
9 & 74 & 1 & 22 & 1 \\
10 & 70 & 1 & 29 & 1 \\
11 & 66 & 1 & 28 & 1 \\
12 & 69 & 3 & 22 & 1
\end{tabular}

Note. MMSE, minimental score exam (Folstein et al., 1975). CDR, clinical dementia rating scale (Hughes et al., 1982): 1 is mild.

TABLE 3: Relative Decrease of Metabolism in PSP Patients Compared to Controls

$$
x(\mathrm{~mm}) \quad y(\mathrm{~mm}) \quad z(\mathrm{~mm}) \quad Z \text { value }
$$

\begin{tabular}{lllll}
\hline L. anterior cingulate (32) & -8 & 28 & 28 & 3.61 \\
& -8 & 8 & 40 & 3.58 \\
L. callosomarginal sulcus (32) & -26 & 8 & 44 & 4.21 \\
L. middle frontal (6/8) & -18 & 20 & 44 & 3.56 \\
L. middle frontal (6) & -34 & 2 & 40 & 4.00 \\
L. precentral (6) & -36 & 2 & 32 & 4.06 \\
Midbrain tegmentum & 0 & -26 & -8 & 3.78 \\
Globus pallidus (internal) & -14 & 4 & 8 & 3.90 \\
R. dorsomedial thalamus & 8 & -12 & 8 & 3.90 \\
L. dorsomedial thalamus & -8 & -14 & 8 & 3.43
\end{tabular}

Note. Metabolic decrease in 10 patients with PSP compared to 8 healthy controls of similar age. Coordinates refer to the stereotactic space of Talairach and Tournoux (1988). Numbers in parentheses refer to Brodmann's areas. Areas reported are included in a SPM thres holded to Z $=3.09(P<0.001)$, further corrected for multiple comparisons. L, left; $\mathrm{R}$, right.

\section{RESULTS}

Our first aim was to determine the precise location of brain metabolic impairment in PSP. When normalized images obtained from PSP patients were compared to those from elderly healthy controls (Table 3 and Fig. 1a, $P$ $<0.001$ and further correction for multiple comparisons), glucose metabolism was reduced in left anterior cingulate (Brodmann area BA32) and callosomarginal sulcus (BA 32), left middle frontal gyrus (BA 6 and 8), left rostrodorsal globus pallidus, dorsomedial nucleus of thalamus (lateral portion), and midbrain tegmen-tum. The left lateralization of cortical involvement corresponded to a bias of population selection, for clinical signs were strictly bilateral in three patients, but mildly predominated on the right body side in six, and on the left side in only one PSP patient. Effectively, SPM with a $Z$ score threshold decreased to 2.58 (Fig. $1 \mathrm{a}, P<0.005$ ) demonstrated a more widespread and bilateral cortical involvement, extending to the left BA 46 (middle frontal gyrus) and to the right cingulate and callosomarginal regions.

Our second aim was to contrast the relative metabolic distribution in prototypes of cortical and subcorti-cal dementia. When PSP images were contrasted to those in SAD (Table 4 and Fig. 1b), relative metabolic 
impairment in PSP was observed in anterior cingulate and along the horizontal portion of the callosomarginal sulcus, and also in adjacent supplementary motor area (SMA, BA 6); in precentral cortex (BA 4/6); in superior, middle, and inferior frontal cortex (BA 9, 8, and 44); in midbrain tegmentum; and in the ventrolateral nucleus of thalamus. The reverse contrast showed that preferential metabolic involvement in SAD patients (compared with PSP) was located in posterior cingulate gyrus (BA 31) and in left parietooccipital sulcus (Table 4 and Fig. 1c, $P$ $<0.001$ and further correction for multiple comparisons), and to a lesser degree in the middle temporal gyrus, the posterior part of the superior temporal gyrus, the inferior parietal gyrus, the superior occipital gyrus, and the left cuneus (Fig. 1c, $P<0.005$ ).

TABLE 4: Contrasted Patterns of Relative Decrease in Metabolism

$$
x(\mathrm{~mm}) \quad y(\mathrm{~mm}) \quad z(\mathrm{~mm}) \quad Z \text { value }
$$

\section{PSP versus SAD}

L. anterior cingulate $(32)$

$\begin{array}{llll}-10 & 2 & 44 & 4.51\end{array}$

L. callosomarginal sulcus (32)

$\begin{array}{llll}-12 & 16 & 44 & 4.11\end{array}$

$\begin{array}{llll}-8 & -14 & 44 & 3.48\end{array}$

R. callosomarginal sulcus (32)

$\begin{array}{llll}-10 & -24 & 44 & 3.26\end{array}$

1420

$\begin{array}{lll}14 & 30 & 32\end{array}$

R. supplementary motor area (6)

$10 \quad 6$

L. precentral (4/6)

$\begin{array}{llll}-34 & -12 & 40 & 4.84\end{array}$

$\begin{array}{llll}-36 & -22 & 40 & 4.24\end{array}$

$\begin{array}{llll}-44 & -14 & 32 & 3.87\end{array}$

R. precentral (4/6)

L. inferior frontal (44)

$46 \quad-6$

32

L. middle frontal (8)

$\begin{array}{lll}-44 & 16 & 20\end{array}$

3.65

L. superior frontal (8/9)

$-46 \quad 12$

$-12 \quad 38$

Midbrain tegmentum

0

$38 \quad 32$

4.65

L. ventrolateral thalamus

$\begin{array}{llll}-16 & -12 & 8 & 4.28\end{array}$

R. ventrolateral thalamus

14

$-14$

8

3.96

\section{SAD versus PSP}

$\begin{array}{lcccc}\text { R. posterior cingulate (31) } & 16 & -56 & 28 & 5.03 \\ \text { L. posterior cingulate (31) } & -12 & -48 & 28 & 3.25 \\ \text { L. parieto-occipital sulcus } & -14 & -70 & 20 & 4.26\end{array}$

Note. Reciprocal metabolic comparison between 12 SAD patients and 10 patients with PSP. Coordinates refer to the stereotactic space of Talairach and Tournoux (1988). Numbers in parentheses refer to Brodmann's areas. All areas reported for PSP are included in a SPM thresholded to $Z=3.09$ ( $P<0.001)$, further corrected for multiple comparisons. L, left; R, right. 

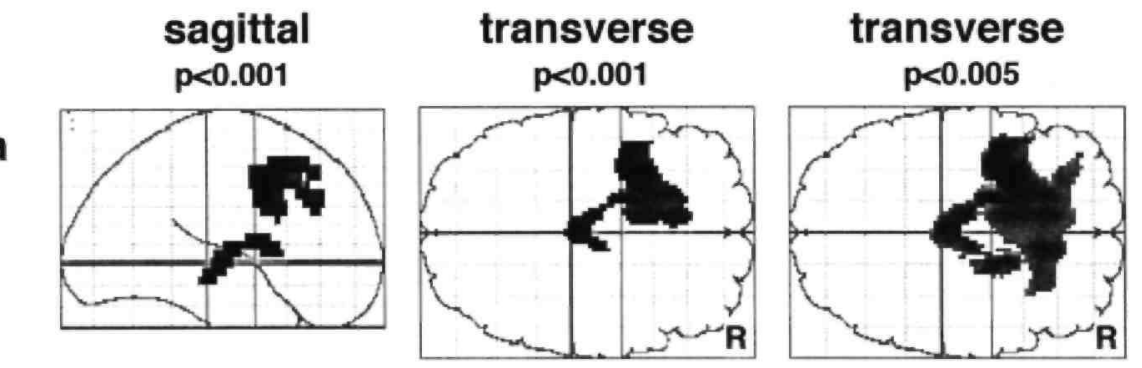

b
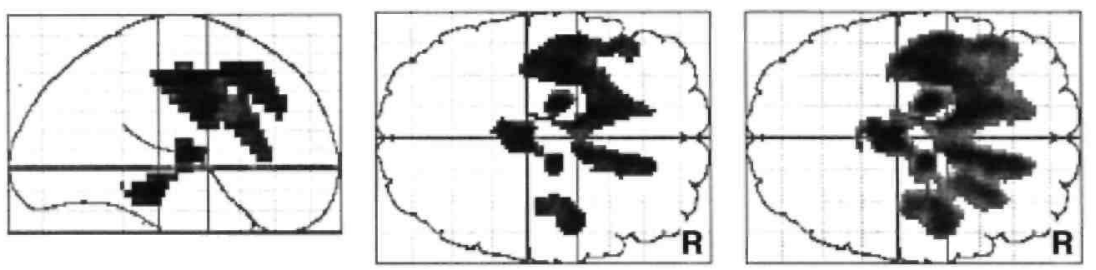

C
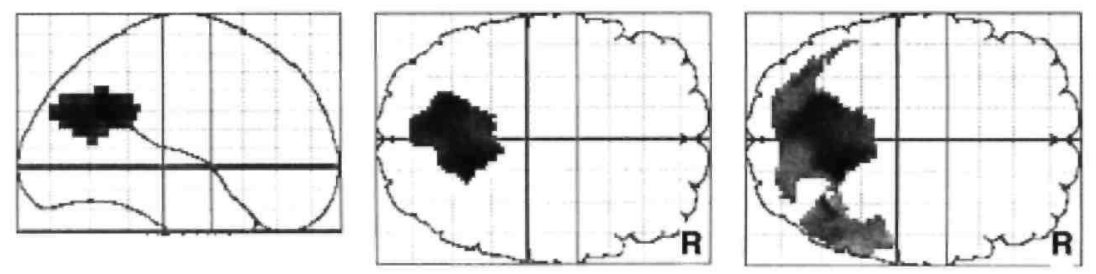

FIG. 1. Statistical parametric maps thresholded to $Z=3.09(P<0.001)$ and to $Z=2.58(P<0.005)$, showing the distribution of brain areas with significant metabolic differences between PSP and controls (a), between PSP and SAD (b), and (reverse contrast) between SAD and PSP (c). Figures are displayed in sagittal and transverse projections into a stereotactic space (Talairach and Tournoux, 1988).

\section{DISCUSSION}

Previous studies using PET and regions of interest analysis in patients suffering from PSP already showed hypometabolism in cortical (and mainly frontal) regions, striatum, thalamus, and brain stem (D'Antona et al., 1985; Leenders et al., 1988; Foster et al., 1988; Goffinet et al., 1989; Blin et al., 1990). Frontal lobe metabolism correlates with performances on tests assessing frontal dysfunction (Blin et al., 1990). Striatal accumulation of $\left[{ }^{18} \mathrm{~F}\right]$ fluorodopa is decreased in PSP (Brooks et al., 1990), while D2 dopaminergic receptors are only mildly reduced (Brooks et al., 1992). Those markers of dopaminergic transmission are not related to locomotor dysfunction, while caudate and thalamic metabolic values correlate with parkinsonian motor score (Blin et al., 1990). Regions of interest analysis, however, did not allow concordant location of metabolic impairment within the frontal lobe: metabolic decrease involved "medial and lateral" frontal cortex (D'Antona et al., 1985), superior and inferior parts of prefrontal regions (Blin et al., 1990), superior anterior frontal (Foster et al., 1988), or the motor/premotor division of the frontal lobe (Goffinet et al., 1989). Moreover, frontal lobe hypometabolism is not specific for PSP, but metabolic differences between degenerative dementias have rarely been explored (Habert et al., 1991; Goto et al., 1993). We used statistical parametric mapping to better localize and characterize the pattern of metabolic impairment in PSP, by comparing brain metabolic images obtained in this prototype of subcortical dementia to those observed in healthy controls of similar age and in Alzheimer's disease with similar dementia severity (taken as prototype of cortical dementia). Standard clinical CT scan showed at most mild atrophy in all patients, but we could not obtain individual magnetic resonance images suitable for localizing more precisely hypometabolic areas or for quantifying focal atrophy (Masucci et al., 1995).

Metabolic comparison between PSP and controls emphasized involvement of subcorticofrontal circuits comprising anterior cingulate, middle frontal cortex, and mediodorsal thalamic nucleus. There are anatomic connections between these different regions (Vogt et al., 1979; Bates and Goldman-Rakic, 1993). They participate in response selection and attention for action, and they play an important role in the initiation, organization, and maintenance of goal-directed behaviors (Pos-ner and Petersen, 1990; Mesulam, 1990; Devinski et al., 1995). Anterior cingulate and prefrontal cortex are activated during neuropsychological tasks such as Stroop paradigm (Pardo et al., 1990) or verbal fluency (Frith et al., 1991), which PSP patients frequently fail. A posterior middle frontal region (close to the precen-tral cortex) affected in PSP may also be part of the frontal eye field (Paus et al., 1993). There is a left lateralization of frontal metabolic impairment in our PSP population, which probably reflects a selection bias, for we observed bilateral involvement of the above-mentioned regions when statistical threshold was lowered. It should be noted that a predominantly left metabolic involvement has already been reported in normal aging (Martin et al., 1991) and inAlzheimer's disease (Lowenstein et al., 1989). 
The comparison of the metabolic pattern observed in PSP to that seen in SAD also demonstrated relative metabolic impairment in anterior cingulate, in calloso-marginal sulcus, and in adjacent SMA, which participate in motor networks (Alexander et al., 1986; Dum and Strick, 1991; Luppino et al., 1991; Paus et al., 1993). In the motor networks, metabolism was also impaired in globus pallidus and midbrain tegmentum, which are consistent sites of neuropathological involvement in PSP, and in the precentral cortex (Brodmann area 4/6), known to be a preferential location of cortical tangles in the disease (Hauw et al., 1990; Daniel et al., 1995). Preferential metabolic impairment of motor/ premotor frontal cortices was already reported in PSP (Goffinet et al., 1989). Finally, statistical analysis in a stereotactic space allowed us to demonstrate a significant reduction of metabolism in the ventral lateral nucleus of thalamus in PSP compared to SAD. Different parts of this nucleus receive inputs from central cerebel-lar nuclei (via the tegmentum) and pallidum and send projections to premotor and supplementary motor areas (Schell and Struck, 1984; Wiesendanger and Wiesen-danger, 1985; Hirai and Jones, 1989).

When SAD metabolic images were compared to those obtained in PSP, glucose metabolism was preferentially impaired in the posterior cingulate cortex of patients suffering from Alzheimer's disease (Minoshima et al., 1994; Reiman et al., 1996). Neuronal degeneration is observed histologically in posterior cingulate cortex of AD patients (Brun and Englund, 1981). Dysfunction of this region probably plays a role in the memory deficits seen in $\mathrm{AD}$, based on its relay position between frontal and parietal associative cortices on the one hand and hippocampal and parahippocampal regions on the other hand (Vogt and Pandya, 1987; Pandya et al., 1981; Goldman-Rakic et al., 1984). In addition to posterior cingulate involvement, our data are consistent with the literature and confirm metabolic impairment in interconnected associative regions, including temporal, parietal, and occipital cortices. These areas subserve complex cognitive processes (which are impaired in AD) and participate in a posterior attentional network (Mesulam, 1990). As in many studies using functional imaging, hippocampal metabolic impairment is not demonstrated inAD (Minoshima et al., 1994; Reiman et al., 1996).

Although many workers in the field emphasize the overlapping between some metabolic, neuropsychologi-cal, or neuropathological characteristics of cortical and subcortical dementias, the present study aimed to differentiate the specific pattern of regional metabolic dysfunction observed in "prototypes" of both types of dementia. The precise location of metabolic impairment obtained with positron emission tomography is difficult to ascertain in subregions of structures such as globus pallidus or thalamic nuclei, but our results in PSP patients demonstrate involvement of the frontosub-cortical circuits described by Alexander and colleagues (1986). More precisely, the data show predominant functional impairment in brain areas participating in motor, anterior executive, and anterior attentional networks in PSP, and they justify viewing the clinical syndrome as a subcorticofrontal dysfunction. This is in contrast to the preferential involvement of posterior cingulate and multimodal associative cortices observed in Alzheimer's disease (Minoshima et al., 1994; Mesu-lam, 1990; Herholz et al., 1993; Reiman et al., 1996).

The differential anterior and posterior metabolic impairment that we observed in PSP and AD could now be used to interpret the respective neuropsychological profiles in terms of variable dysfunction of cognitive networks.

\section{ACKNOWLEDGMENTS}

This work was supported by grants from the Belgian National Fund for Scientific Research (FNRS) and the Fondation Medicale Reine Elisabeth. E. Salmon is Senior ResearchAssistant at FNRS.

\section{REFERENCES}

Albert, M. L., Feldman, R. G., and Willis, A. L. 1974. The 'subcortical dementia' of progressive supranuclear palsy. J. Neurol. Neurosurg. Psychiatry 37:121-130.

Alexander, G. E., DeLong, M. R., and Strick, P. L. 1986. Parallel organisation of functionally segregated circuits linking basal ganglia and cortex. Annu. Rev. Neurosci. 9:357-381.

American Psychiatric Association. 1987. Diagnostic and Statistical Manual of Mental Disorders, 3rd ed. rev. Am. Psychiatr. Assoc., Washington, DC.

Bates, J. F., and Goldman-Rakic, P. S. 1993. Prefrontal connections of medial motor areas in the rhesus monkey. J. Comp. Neurol. 336:211228 .

Blin, J., Baron, J. C., Dubois, P., et al. 1990. Positron emission tomography in progressive supranuclear palsy. Arch. Neurol. 47: 747-752.

Brooks, D. J., Ibanez, V., Sawle, G. V., et al. 1990. Differing patterns of striatal ${ }^{18}$ F-dopa uptake in Parkinson's disease, multiple system atrophy and progressive supranuclear palsy. Ann. Neurol. 28:547-555.

Brooks, D. J., Ibanez, V., Sawle, G. V., et al. 1992. Striatal D2 receptor status in Parkinson's disease, striatonigral degeneration, and progressive supranuclear palsy, measured with ${ }^{11}$ C-raclopride and PET. Ann. Neurol. 31:184-192.

Brun, A., and Englund, E. 1981. Regional pattern of degeneration in Alzheimer's disease: Neuronal loss and histopathological grading. Histopathology 5:549-564.

Collins, S. J., Ahlskog, J. E., Parisi, J. E., and Maraganore, D. M. 1995. Progressive supranuclear palsy: Neuropathologically based 
Published in: Neuroimage (1997), vol. 5, iss. 3, pp. 173-178

Status: Postprint (Author's version)

diagnostic clinical criteria. J. Neurol. Neurosurg. Psychiatry 58:167-173.

Daniel, S. E., de Bruin, V. M. S., and Lees,A. J. 1995. The clinical and pathological spectrum of Steele-Richardson-Olszewski syndrome (progressive supranuclear palsy): A reappraisal. Brain 118:759-770.

D’Antona, R., Baron, J. C., Samson, Y., et al. 1985. Subcortical dementia: Frontal cortex hypometabolism detected by positron emission tomography in patients with progressive supranuclear palsy. Brain 108:785-800.

Devinsky, O., Morrel, M. J., and Vogt, B. A. 1995. Contribution of anterior cingulate cortex to behaviour. Brain 118:279-306.

Dum, R. P., and Strick, P. L. 1991. The origin of corticospinal projections from the premotor areas in the frontal lobe. J. Neurosci. 11:667689.

Folstein, M. F., Folstein, S. E., and McHugh, P. R. 1975. Mini-mental state: A practical method for grading the cognitive state of patients for the clinician. J. Psychiatr. Res. 12:189-198.

Foster, N. L., Gilman, S., Berent, S., Morin, E. M., Brown, M. B., and Koeppe, R. A. 1988. Cerebral hypometabolism in progressive supranuclear palsy studied with positron emission tomography. Ann. Neurol. 24:399-406.

Friston, K., Holmes, A. P., Worsley, K. J., Poline, J. B., Frith, C. D., and Frackowiak, R. S. J. 1995. Statistical parametric maps in functional imaging: A general linear approach. Hum. Brain Map. 2:189-210.

Friston, K. J., Frith, C. D., Liddle, P. F., and Frackowiak, R. S. J. 1991. Comparing functional (PET) images: The assessment of significant change. J. Cereb. Blood Flow Metab. 11:690-699.

Frith, C. D., Friston, K. J., Liddle, P. F., and Frackowiak, R. S. J. 1991. Willed action and the prefrontal action in man: A study with PET. Proc. R. Soc. London Biol. 244:241-246.

Gearing, M., Olson, D. A., Watts, R. L., and Mirra, S. S. 1994. Progressive supranuclear palsy: Neuropathologic and clinical heterogeneity. Neurology 44:1015-1024.

Goffinet, A. M., De Volder, A. G., Gillain, C., et al. 1989. Positron emission tomography demonstrates frontal lobe hypometabolism in progressive supranuclear palsy. Ann. Neurol. 25:131-139.

Goldman-Rakic, P. S., Selemon, L.D., and Schwartz, M. L. 1984. Dual pathways connecting the dorsolateral prefrontal cortex with the hippocampal formation and parahippocampal cortex in the rhesus monkey. Neuroscience 12:719-743.

Goto, I., Taniwaki, T., Hosokawa, S., Otsuka, M., Ichiya, Y., and Ichimiya,A. 1993. Positron emission tomographic (PET) studies in dementia. J. Neurol. Sci. 114:1-6.

Habert, M. O., Spampinato, U., Mas, J. L., et al. 1991. A comparative technetium 99m hexamethylpropylene amine oxime SPET study in different types of dementia. J. Nucl. Med. 18:3-11.

Hauw, J. J., Daniel, S. E., Dickson, D., et al. 1990. Preliminary NINDS neuropathologic criteria for Steele-Richardson-Olszewski syndrome (progressive supranuclear palsy). Neurosci. Lett. 119:182- 186.

Herholz, K., Perani, D., Salmon, E., Franck, G., Fazio, F., Heiss, W. D., and Comar, D. 1993. Comparability of FDG PET studies in probable Alzheimer's disease. $J$. Nucl. Med. 34:1460-1466.

Hirai, T., and Jones, E. G. 1989. A new parcellation of the thalamus on the basis of histochemical staining. Brain Res. Rev. 14:1-34.

Hughes, C. P., Berg, L., Danziger, W. L., Coben, L. A., and Martin, R. L. 1982. A new clinical scale for the staging of dementia. Br. J Psychiatry 140:566-572.

Leenders, K. L., Frackowiak, R. S. J., and Lees, A. J. 1988. Steele- Richardson-Olszewski syndrome: Brain energy metabolism, blood flow, and fluorodopa uptake measured by positron emission tomography. Brain 111:615-630.

Litvan, I., Agid, Y., and Calne, D. 1996. Clinical research criteria for the diagnosis of progressive supranuclear palsy (Steele-Richard-sonOlszewski syndrome). Neurology 47:1-9.

Lowenstein, D., Barker, W., Chang, J., et al. 1989. Predominant left hemisphere metabolic dysfunction in dementia. Arch. Neurol. 46:146152.

Luppino, G., Matelli, M., Camarda, R. M., Gallese, V., and Rizzolatti, G. 1991. Multiple representations of body movements in mesial area 6 and the adjacent cingulate cortex: An intracortical micro-stimulation study in the macaque monkey. J. Comp. Neurol. 311:463-482.

Martin, A. J., Friston, K. J., Colebatch, J. G., and Frackowiak, R. S. J. 1991. Decreases in regional cerebral blood flow with normal aging. J. Cereb. Blood Flow Metab. 11:684-689.

Masucci, E. F., Borts, F. T., Perl, S. M., Wener, L., Schwankhaus, J., and Kurtzke, J. F. 1995. MR versus CT in progressive supranuclear palsy. Comput. Med. Imaging Graph. 19:361-368.

McKhann, G., Drachman, D., Folstein, M., Katzman, R., Price, D., and Stadlan, E. M. 1984. Clinical diagnosis of Alzheimer's disease: Report of the NINCDS-ADRDA work group. Neurology 34:939-944.

Mesulam, M. M. 1990. Large-scale neurocognitive networks and distributed processing for attention, language, and memory. Ann. Neurol. 28:597-613

Minoshima, S., Foster, N. L., and Kuhl, D. E. 1994. Posterior cingulate cortex in Alzheimer's disease. Lancet 344:895.

Pandya, D. N., Van Hoesen, G. W., and Mesulam, M.-M. 1981. Efferent connections of the cingulate gyrus in the rhesus monkey. Exp. Brain Res. 42:319-330.

Pardo, J. V., Pardo, P. J., Janer, K. W., and Raichle, M. E. 1990. The anterior cingulate cortex mediates processing selection in the Stroop attentional conflict paradigm. Proc. Natl. Acad. Sci. USA 87:256-259. 
Parkes, J. D., Zilka, K. J., Marsden, P., Baxter, R. C. H., and Knill-Jones, R. P. 1970.Amantadine dosage in treatment of Parkinson's disease. Lancet i: $1130-1133$

Paus, T., Petrides, M., Evans, A. C., and Meyer, E. 1993. Role of the human anterior cingulate cortex in the control of oculomotor, manual, and speech responses: A positron emission tomography study. J. Neurophysiol. 2:453-469.

Phelps, M. E., Huang, S. E., Hoffman, E. J., Selin, S. C., Sokoloff, L., and Kuhl, D. E. 1979. Tomographic measurement of local cerebral metabolic rate in humans with (F-18)-2-fluoro-2-deoxyglucose: Validation of method. Ann. Neurol. 6:371-388.

Pillon, B., Dubois, B., Ploska, A., and Agid, Y. 1991. Severity and specificity of cognitive impairment in Alzheimer's, Huntington's, and Parkinson's diseases and progressive supranuclear palsy. Neurology 41:634-643.

Posner, M. I., and Petersen, S. E. 1990. The attentional system of the human brain. Annu. Rev. Neurosci. 13:25-42.

Reiman, E. M., Caselli, R. J., Yun, L. S., et al. 1996. Preclinical evidence of Alzheimer's disease in persons homozygous for the epsilon4 allele for apolipoprotein E. N. Engl. J. Med. 334:752-758.

Schell, G. R., and Strick, P. L. 1984. The origin of thalamic inputs to the arcuate premotor and supplementary motor areas. J. Neurosci. 4:539-560.

Steele, J. C., Richardson, J. C., and Olszewski, J. 1964. Progressive supranuclear palsy. Arch. Neurol. (Chicago) 10:333-359.

Talairach, J., and Tournoux, P. 1988. Co-Planar Stereotactic Atlas of the Human Brain. Thieme, Stuttgart. Valenstein, E., Bowers, D., Verfaellie, M., Heilman, K. M., Day, A., and Watson, R. T. 1987. Retrosplenial amnesia. Brain 110:1631-1646.

Vogt, B.A., and Pandya, D. N. J. 1987. Cingulate cortex of the rhesus monkey: Cortical afferents. J. Comp. Neurol. 262:271-289.

Vogt, B. A., Rosene, D. L., and Pandya, D. N. 1979. Thalamic and cortical afferents differentiate anterior from posterior cingulated cortex in the monkey. Science 204:205-207.

Whitehouse, P. J. 1986. The concept of subcortical and cortical dementia: Another look. Ann. Neurol. 19:1-6.

Wiesendanger, R., and Wiesendanger, M. 1985. The thalamic connections with medial area 6 (supplementary motor cortex) in the monkey (Macaca fascicularis). Exp. Brain Res. 59:91-104. 